\title{
THE QUOTIENT SEMIGROUP OF A SEMIGROUP THAT IS A SEMILATTICE OF GROUPS $\dagger$
}

\author{
by F. R. McMORRIS
}

(Received 13 October, 1969)

1. Introduction. Let $Q(S)$ denote the maximal right quotient semigroup of the semigroup $S$ as defined in [4]. In this paper, we initiate a study of $Q(S)$ when $S$ is a semilattice of groups. A structure theorem for such semigroups is given by Theorem 4.11 of [2].

We prove that if $S$ is a semilattice of groups, then so is $Q(S)$. In the process of showing this, we look at how right $S$-homomorphisms act on the groups making up $S$. In particular, a right $S$-homomorphism takes a group into a group with a lower index, and then maps this group one-to-one and onto itself.

If the set of idempotents of $S$ forms a chain, then $Q(S)$ and $S$ have exactly the same idempotents, and $Q(S)$ is just $S$ union the group of units of $Q(S)$. If $S$ is itself a chain, then $S=Q(S)$

2. Preliminaries. Terminology throughout this note will be as found in [2] and [4].

Definition 2.1. Let $S$ be a subsemigroup of $T$. Then $T$ is a right quotient semigroup of $S$ if and only if, for any three elements $t_{1}, t_{2}, t \in T$ with $t_{1} \neq t_{2}$, there exists an element $s \in S$ such that $t_{1} s \neq t_{2} s$ and $t s \in S$.

Definition 2.2. If $D$ is a right ideal of $S$, then $D$ is said to be dense if and only if $S$ is a right quotient semigroup of $D$. The set of all dense right ideals of $S$ will be denoted by $S^{\Delta}$.

Let us recall that $Q(S)=H_{S} \mid \equiv$, where $H_{S}=\bigcup\left\{\operatorname{Hom}_{S}(D, S): D \in S^{\Delta}\right\}$ and $\equiv$ is the congruence defined by $f_{1} \equiv f_{2}$ if and only if $f_{1}$ agrees with $f_{2}$ on some dense right ideal contained in the intersection of their domains. We denote the domain of $f \in H_{S}$ by $D_{f}$, and the equivalence class containing $f$ by $[f]$. Thus $[f]=[g]$ if and only if $f=g$ on some $D \in S^{\Delta}$ with $D \subseteq D_{f} \cap D_{\theta}$. $S$ is considered as a subsemigroup of $Q(S)$ under the identification $x \rightarrow\left[x_{l}\right]$, where $x_{l}$ is the left multiplication by $x$.

From now on, we shall let $S$ be a semigroup with 0 and 1 that is a semilattice $Y$ of groups $G_{\alpha}(\alpha \in Y)$, where $Y$ is a semilattice order isomorphic to $E(S)$, the set of idempotents of $S$. Let $e_{\alpha}$ be the identity of the group $G_{\alpha}$. The zero and identity of $Y$ will also be denoted by 0 and 1 . We recall that $S=\bigcup\left\{G_{\alpha}: \alpha \in Y\right\}$ with $G_{\alpha} \cap G_{\beta}=\emptyset$ if $\alpha \neq \beta$, and $G_{\alpha} G_{\beta} \subseteq G_{\alpha \beta}$.

By [2, exercise 2, p. 129], every one-sided ideal of $S$ is two-sided. Thus $D \in S^{\Delta}$ if and only if, for any two elements $x_{1}, x_{2} \in S$ with $x_{1} \neq x_{2}$, there exists an element $d \in D$ such that $x_{1} d \neq x_{2} d$.

3. In this section we show that $Q(S)$ is also a semilattice of groups. We recall that a semigroup $T$ is regular if and only if, for every element $x \in T$, there exists an element $y \in T$ such that $x y x=x$.

† This is a portion of the author's doctoral dissertation written at the University of Wisconsin-Milwaukee under the direction of Professor R. L. Gantos. 
PROPOSITION 3.1 ([2], pp. 128-129). A semigroup $T$ is regular with idempotents in the centre of $T$ if and only if $T$ is a semilattice of groups.

We shall show that $Q(S)$ is regular and has central idempotents, but first we need the following lemmas.

Lemma 3.2. If $D$ is an ideal of $S$, then $D$ is a semilattice of groups.

Proof. We assert that $D$ is a semilattice $X_{D}$ of groups $G_{\beta}\left(\beta \in X_{D}\right)$, where $X_{D}$ is an ideal of $Y$. Let $d \in D$; then $d \in G_{\beta}$ for some $\beta \in Y$, and thus there exists an element $d^{-1} \in G_{\beta}$ such that $d d^{-1}=d^{-1} d=e_{\beta}$. Since $D$ is an ideal, we have $e_{\beta} \in D$, and it follows that $G_{\beta} \subseteq D$. Set $X_{D}=\left\{\beta \in Y: e_{\beta} \in D\right\}$ and let $\beta \in X_{D}, \alpha \in Y$. Since $e_{\beta} \in D$, we have $e_{\alpha} e_{\beta} \in D$. Thus $e_{\alpha} e_{\beta}=e_{\alpha \beta}$ implies that $\alpha \beta \in X_{D}$. Hence $X_{D}$ is an ideal of $Y$ and $D$ is a semilattice $X_{D}$ of groups $G_{\beta}\left(\beta \in X_{D}\right)$.

We shall let $E(D)=\left\{e_{\beta} \in E(S): e_{\beta} \in D\right\}$. Thus $E(D)$ is order isomorphic to $X_{D}$ under the correspondence $\beta \rightarrow e_{\beta}$. If $f \in H_{S}$, let $E_{f}=\left\{e_{\alpha} \in E(S): e_{\alpha} \in D_{f}\right\}$.

Lемма 3.3. Let $f \in H_{S}$. If $J$ is an ideal of $S$ such that $J \subseteq D_{f}$, then $f(J) \subseteq J$. In particular, $f\left(D_{f}\right) \subseteq D_{f}$.

Proof. Let $x \in J$; then $x \in G_{\alpha}$ for some $\alpha \in X_{J}$, and $f(x)=f\left(x e_{\alpha}\right)=f(x) e_{\alpha} \in J$.

REMARK 3.4. If $e_{\alpha} \leqq e_{\beta}(\alpha \leqq \beta)$, then $e_{\beta} x=x$ for all $x \in G_{\alpha}$ (for $e_{\beta} x=e_{\beta}\left(e_{\alpha} x\right)=\left(e_{\beta} e_{\alpha}\right) x=$ $\left.e_{\alpha} x=x\right)$.

LEмma 3.5. Let $f \in H_{S}$; then for all $e_{\beta} \in E_{f}$, there exists a unique $e_{\gamma} \in E_{f}$, with $e_{\gamma} \leqq e_{\beta}$, such that $f\left(G_{\beta}\right) \subseteq G_{\gamma}$. Also, $f$ restricted to $G_{\gamma}$ is a one-to-one mapping of $G_{\gamma}$ onto $G_{\gamma}$.

Proof. Let $e_{\beta} \in E_{f}$, and consider the element $f\left(e_{\beta}\right)$. From 3.2 and 3.3, we have that $f\left(e_{\beta}\right) \in G_{\gamma}$ for some $e_{\gamma} \in E_{\gamma}$. Now $f\left(e_{\beta}\right)=f\left(e_{\beta} e_{\beta}\right)=f\left(e_{\beta}\right) e_{\beta} \in G_{\gamma} G_{\beta} \subseteq G_{\gamma \beta}$. Hence $f\left(e_{\beta}\right) \in G_{\gamma \beta} \cap G_{\gamma}$ and thus $G_{\gamma \beta}=G_{\gamma}$, which implies that $\gamma \beta=\gamma$. Therefore $\gamma \leqq \beta\left(e_{\gamma} \leqq e_{\beta}\right)$. Now let $x \in G_{\beta}$; then $f(x)=f\left(e_{\beta} x\right)=f\left(e_{\beta}\right) x \in G_{\gamma} G_{\beta} \subseteq G_{\gamma \beta}=G_{\gamma}$. Thus we have $f\left(G_{\beta}\right) \subseteq G_{\gamma}$. It is clear that $e_{\gamma}$ is unique since $S$ is the disjoint union of the groups $G_{\alpha}(\alpha \in Y)$.

If $y \in G_{\gamma}$, then we have $e_{\beta} y=y$, by 3.4. Thus we have $f(y)=f\left(e_{\beta} y\right)=f\left(e_{\beta}\right) y \in G_{\gamma} G_{\gamma} \subseteq G_{\gamma}$. Hence $f\left(G_{\gamma}\right) \subseteq G_{\gamma}$. Finally it remains to show that $f$ takes $G_{\gamma}$ one-to-one and onto itself. Assume that $y, z \in G_{y}$, with $f(y)=f(z)$; then $f(y)=f\left(e_{y} y\right)=f\left(e_{\gamma}\right) y=f\left(e_{\gamma}\right) z=f\left(e_{\gamma} z\right)=f(z)$. Cancelling $f\left(e_{\gamma}\right)$, we have that $y=z$. Now let $w \in G_{\gamma}$; then there exists an element $u \in G_{\gamma}$ such that $f\left(e_{\gamma}\right) u=w$. But $f\left(e_{\gamma}\right) u=f(u)$, and this completes the proof.

RemarK 3.6. Suppose that $f \in H_{s}$ and $e_{\beta} \in E_{f}$. Let $e_{\gamma}$ be as given in 3.5. Then $f f$ is also a one-to-one mapping of $G_{\gamma}$ onto $G_{\gamma}$.

THEOREM 3.7. $Q(S)$ is a regular semigroup.

Proof. Let $[f] \in Q(S)$. We shall define a mapping $g \in H_{S}$ such that $[f][g][f]=[f]$. Let $x \in D_{f}$, so that $x \in G_{\beta}$ for some $e_{\beta} \in E_{f}$. Let $e_{\gamma}$ be as in 3.5. Then from 3.6, we see that there exists a unique $y \in G_{y}$ such that $f(y)=f(x)$. Define the mapping $g: D_{f} \rightarrow S$ by $g(x)=y$. We assert that $g$ is a right $S$-homomorphism. Assume that $x \in D_{f}$ with $x \in G_{\beta}$, and $s \in S$ with $s \in G_{\alpha}$. Let $y \in G_{\gamma}$ be as chosen above. Set $z=g(x s)$. Since $f(x s)=f(x) s \in G_{\gamma} G_{\alpha} \subseteq G_{\gamma \alpha}$, it follows 
that $z \in G_{y \alpha}$ with $f f(z)=f(x s)$. Now $f f(z)=f(x s)=f(x) s=(f f(y)) s=f f(y s)$. Since $z, y s \in G_{y \alpha}$ and $f f$ is one-to-one on $G_{y \alpha}$, we have $z=y s$; that is, $g(x s)=g(x) s$.

We show that $[f][g][f]=[f]$ by proving that $f g f$ agrees with $f$ on $D_{f}$. Again let $x \in D_{f}$ with $x \in G_{\beta}$, and $G_{y}$ be as above. Now $f g f(x)=f(u)$, where $u=g(f(x)) \in G_{y}$, and $f(u)=f(f(x))$. Since $f$ is one-to-one on $G$ and $f(u), f(x) \in G_{y}$, we have $f(u)=f(x)$; that is, $f g f(x)=f(x)$.

We recall from 3.1 that every idempotent of $S$ is in the center of $S$. This fact will be used throughout the proofs of the following lemmas.

Lemma 3.8. Let $f \in H_{s}$. If $f f=f$ on some ideal $J$ with $J \subseteq D_{f}$, then $f(e) \in E(J)$ for all $e \in E(J)$.

Proof. Let $e \in E(J)$; then $f(e)=f f(e)=f f(e e)=f(f(e) e)=f(e f(e))=f(e) f(e)$.

Lemma 3.9. Let $f \in H_{s}$; then $f f=f$ on an ideal $J$ with $J \subseteq D_{f}$ if and only if $f(x y)=f(x) f(y)$ for all $x, y \in J$.

Proof. Assume that $f f=f$ on $J \subseteq D_{f}$, and let $x, y \in J$ with $x \in G_{\alpha}$ and $y \in G_{\beta}$. Applying 3.8, we have

$$
\begin{aligned}
f(x y) & =f f(x y)=f f\left(e_{\alpha} x e_{\beta} y\right)=f f\left(e_{\alpha} e_{\beta} x y\right) \\
& =\left(f f\left(e_{\alpha} e_{\beta}\right)\right) x y=\left(f\left(f\left(e_{\alpha}\right) e_{\beta}\right)\right) x y=\left(f\left(e_{\alpha} f\left(e_{\beta}\right)\right)\right) x y \\
& =f\left(e_{\alpha}\right) f\left(e_{\beta}\right) x y=f\left(e_{\alpha}\right) x f\left(e_{\beta}\right) y=f(x) f(y) .
\end{aligned}
$$

For the converse, let $z \in J$ with $z \in G_{y}$. Then

$$
f(z)=f\left(e_{\gamma} z\right)=f\left(e_{\gamma}\right) f(z)=f\left(e_{\gamma} f(z)\right)=f\left(f(z) e_{y}\right)=f(f(z))=f f(z) .
$$

Proposition 3.10 (2.33 of [4]). If $T$ is a right quotient semigroup of $S$, then an element of $T$ commutes with every element of $S$ if and only if it is in the centre of $T$.

Proposition 3.11. The idempotents of $Q(S)$ are in the center of $Q(S)$.

Proof. We need only show that if $[f]$ is an idempotent of $Q(S)$, then $[f] x=x[f]$ for all $x \in S$. That is we must show that the mappings $x_{1} f$ and $f x_{1}$ agree on some dense ideal of $S$. Assume that $f f=f$ on $D \in S^{\Delta}$, with $D \subseteq D_{f}$. Set $D^{*}=D \cap D_{f x_{1}}$ and let $d \in D^{*}$ with $d \in G_{\alpha}$. Applying 3.8 and 3.9 , we have

$$
\begin{aligned}
\left(f x_{l}\right)(d) & =f(x d)=f\left(x e_{\alpha} d\right)=f\left(x e_{\alpha}\right) f(d)=f\left(e_{\alpha}\right) x f(d) \\
& =x f\left(e_{\alpha}\right) f(d)=x f\left(e_{\alpha} d\right)=x f(d)=\left(x_{l} f\right)(d) .
\end{aligned}
$$

Hence $x_{l} f=f x_{l}$ on $D^{*} \in S^{\Delta}$.

THEOREM 3.12. $Q(S)$ is a semilattice of groups.

Proof. From 3.7 and $3.11, Q(S)$ is a regular semigroup with central idempotents. Hence, by $3.1, Q(S)$ is a semilattice of groups. 
From 3.1, a commutative semigroup is regular if and only if it is a semilattice of groups. The following example is a commutative example in which $Q(T)$ is regular but $T$ is not. Hence the converse to 3.12 is not necessarily true.

EXAMPLE 3.13. Let $T$ be the infinite cyclic semigroup generated by the element $a$, with 0 and 1 adjoined; that is, $T=\left\{a, a^{2}, a^{3}, \ldots\right\} \cup 0 \cup 1$. Thus $T$ is a commutative semigroup that is not regular. Every ideal of $T$ is of the form $\left\{a^{k}, a^{k+1}, \ldots\right\} \cup 0$ where $k \geqq 1$. It can be shown that every ideal of $T$ is dense, and every $f \in H_{T}$ is one-to-one. Let $f^{\prime}$ be the inverse mapping of $f$. Hence $f^{\prime}$ is a right $S$-homomorphism from $f\left(D_{f}\right) \in T^{\Delta}$ into $T$ such that $f^{\prime} f=f$ on $D_{f}$. Therefore $[f]\left[f^{\prime}\right][f]=[f]$, which implies that $Q(T)$ is a regular semigroup. $Q(T)$ is commutative, by 2.35 of [4].

4. Throughout this section, we shall assume that $E(S)$ is a chain.

Proposition 4.1. Let $G_{1}$ denote the group of units of $S$. If $D \in S^{\Delta}$, then $D=S$ or $D=S-G_{1}$, where $S-G_{1}=\left\{x \in S: x \notin G_{1}\right\}$.

Proof. Assume that $D \in S^{\Delta}$ with $D \notin S$. From $3.2, D$ is a semilattice $X_{D}$ of groups, where $X_{D}$ is isomorphic to $E(D)$. Thus we need only show that $E(D)=E(S)-\{1\}$. Let $e_{\beta} \in E(S)-E(D)$. It is easy to verify that $e_{\alpha} \leqq e_{\beta}$ for all $e_{\alpha} \in E(D)$. Hence, from 3.4, $1 d=d=e_{\beta} d$ for all $d \in D$. Since $D \in S^{\Delta}$, this implies that $1=e_{\beta}$.

Lemma 4.2. Let $[f] \in Q(S)$ and $D=S-G_{1}$. If $f f=f$ on $D$ and $e_{\alpha}, e_{\beta} \in E(D)-f(D)$, then $f\left(e_{\alpha}\right)=f\left(e_{\beta}\right)$.

Proof. By 4.1, we have $D \subseteq D_{f}$. Also $f\left(e_{\alpha}\right), f\left(e_{\beta}\right) \in E(D)$, from 3.8. Assume that $e_{\alpha} \leqq e_{\beta}$. We assert that $f\left(e_{\beta}\right)<e_{\alpha}$. If $e_{\alpha} \leqq f\left(e_{\beta}\right)$, then $e_{\alpha}=f\left(e_{\beta}\right) e_{\alpha}=f\left(e_{\beta} e_{\alpha}\right)=f\left(e_{\alpha}\right)$, which contradicts the fact that $e_{\alpha} \notin f(D)$. Hence $f\left(e_{\beta}\right)=f\left(e_{\beta}\right) e_{\alpha}=f\left(e_{\beta} e_{\alpha}\right)=f\left(e_{\alpha}\right)$.

THEOREM 4.3. The idempotents of $S$ and $Q(S)$ are identical.

Proof. Let $E(Q)$ denote the set of idempotents of $Q(S)$. There are two cases: $S-G_{1} \in S^{\Delta}$ or $S-G_{1} \notin S^{\Delta}$.

Assume that $S-G_{1} \notin S^{\Delta}$ and let $[f] \in Q(S)$. Then $f \in \operatorname{Hom}_{s}(S, S)$ and hence $[f]=$ $\left[(f(1))_{l}\right]=f(1) \in S$. Therefore $S=Q(S)$.

Now let $D=S-G_{1}$ and suppose that $D \in S^{\Delta}$. If $[f] \in E(Q)$, then $f f=f$ on $D$. From 3.3, $f(D) \subseteq D$. Assume that $f(D)=D$. We claim that $f=1_{D}$, where $1_{D}$ is the identity map on $D$. If $d \in D$, then there exists an element $x \in D$ such that $f(x)=d$. Thus $f(d)=f f(x)=f(x)=d$. In [4] it was shown that $\left[1_{D}\right]=1$. Therefore $[f]=\left[1_{D}\right]=1 \in E(S)$.

Let $f(D) \nsubseteq D$. Since $f(D)$ is an ideal of $S, 3.2$ implies that there exists an element $e_{\alpha} \in E(D)-f(D)$. Set $e=f\left(e_{\alpha}\right)$; then $e \in E(S)$. Let $d \in D$ with $d \in G_{\beta}$. If $e_{\beta} \leqq e_{\alpha}$, then $f(d)=$ $f\left(e_{\alpha} d\right)=f\left(e_{\alpha}\right) d=e d$. If $e_{\alpha}<e_{\beta}$, then $e_{\beta} \in E(D)-f(D)$ and we have $f(d)=f\left(e_{\beta} d\right)=f\left(e_{\beta}\right) d=$ $f\left(e_{a}\right) d=e d$, by 4.2 . Hence $[f]=\left[e_{t}\right]=e \in E(S)$.

Theorem 16 of [1] states that, if $S$ is a semilattice $\left(G_{\gamma}=\left\{e_{\gamma}\right\}\right.$ for all $\left.\gamma \in Y\right)$, then so is $Q(S)$. The following corollary then follows. 
Corollary 4.4. If $S$ is a chain, then $S=Q(S)$.

On page 45 of [3], it is shown that if $R$ is a Boolean ring ( $a a=a$ for all $a \in R$ ), then its Dedekind-MacNeille completion is isomorphic over $R$ to the maximal right quotient ring of $R$. An analogous theorem is not true for semilattices: that is, if $S$ is a non-complete chain, then $S=Q(S)$, which is properly contained in its completion.

If $T$ is a semigroup, then $E(T)$ is dually well-ordered if every non-empty subset of $E(T)$ has a greatest element in the set.

THEOREM 4.5. If $T$ is a regular semigroup such that $E(T)$ is dually well-ordered, then $T=Q(T)$.

Proof. We first show that every right ideal is generated by an idempotent. Let $R$ be a right ideal of $T$. Since $T$ is regular, we have $R \cap E(T) \neq \emptyset$. Let $e$ be the greatest idempotent of $T$ contained in $R$. Clearly $e T \subseteq R$. If $x \in R$, then there exists an element $x^{\prime} \in T$ such that $x x^{\prime} x=x$ and $x x^{\prime} \in E(T)$. Now $x x^{\prime} \in R \cap E(T)$, so that $x x^{\prime} \leqq e$. Thus $x=\left(x x^{\prime}\right) x=e\left(x x^{\prime}\right) x \in e T$. Hence $e T=R$.

Now let $f \in H_{T}$; then $D_{f}=i T$, where $i \in E(T)$. We have $f(i y)=f(i i y)=f(i) i y$ for all iyeiT. By 2.31 of [4], $T=Q(T)$.

We shall now write $Q(S)$ as the semilattice $I$ of groups $H_{\alpha}(\alpha \in I)$, where $I$ is isomorphic to $E(Q)$. Note that we may assume that $Y \subseteq I$ and $G_{\alpha} \subseteq H_{\alpha}$ for all $\alpha \in Y$.

LEMMA 4.6. If $\alpha \in Y$ with $\alpha \neq 1$, then $G_{\alpha}=H_{\alpha}$.

Proof. Let $[f] \in H_{\alpha}$, where $\alpha \in Y$ with $\alpha \neq 1$. Thus $e_{\alpha} \neq 1$. Set $e=e_{\alpha}$; then $[f] e=[f]$, which implies that $f e_{l}=f$ on some $D \in S^{\Delta}$, with $D \subseteq D_{f}$. Since $D=S$ or $D=S-G_{1}$, we have $e \in D$. Hence $\left(f e_{l}\right)(d)=f(e d)=f(e) d$. Therefore $[f]=[f] e=\left[(f(e))_{l}\right] \in S$, and thus $[f] \in G_{a}$.

THEOREM 4.7. $Q(S)=\left(\bigcup_{\alpha \neq 1} G_{\alpha}\right) \cup H_{1}$.

Proof. By 4.3, $Y=I$ and hence the result follows from 4.6.

5. For the remainder of this paper, let $T$ be a semigroup with 0 and 1 . A right ideal $R$ of $T$ is said to be minimal if $R \neq 0$ and if $K$ is a right ideal of $T$ with $0 \neq K \subseteq R$, then $K=R . T$ is said to satisfy the minimum condition on right ideals if every non-empty set of right ideals of $T$ has a minimal member.

Proposition 5.1. If T has a minimal dense right ideal, then it is unique.

Proof. This follows from the fact that the intersection of two dense ideals is a dense ideal.

Assume that $T$ has a minimal dense right ideal $D$, and let $f, g \in \operatorname{Hom}_{T}(D, T)$. Let $f g$ be the composition map with domain $g^{-1} D=\{x \in D: g(x) \in D\}$. By 2.14 of $[4], g^{-1} D \in T^{\Delta}$, which implies that $g^{-1} D=D$ since $D$ is minimal. Thus $\operatorname{Hom}_{T}(D, T)$ is a semigroup under this operation.

THEOREM 5.2. If $T$ has a minimal dense right ideal $D$, then $Q(T)$ is isomorphic to $\operatorname{Hom}_{T}(D, T)$. 
Proof. Define the mapping $\mu: Q(T) \rightarrow \operatorname{Hom}_{T}(D, T)$ by $\mu([f])=\left.f\right|_{D}$, where $\left.f\right|_{D}$ is the restriction of $f$ to $D . \mu$ is an isomorphism.

COROllary 5.3. Let $T$ satisfy the minimum condition on right ideals, and let $D$ be the unique minimal dense right ideal of $T$. Then $Q(T)$ is isomorphic to $\operatorname{Hom}_{T}(D, T)$.

COROLlaRY 5.4. Assume that $S$ is a semilattice of groups and $E(S)$ is a finite set. Let $D^{*}$ be the intersection of all the dense ideals of $S$. Then $Q(S)$ is isomorphic to $\operatorname{Hom}_{S}\left(D^{*}, S\right)$.

\section{REFERENCES}

1. P. Berthiaume, The injective envelope of $S$-sets, Canad. Math. Bull. 10 (1967), 261-273.

2. A. H. Clifford and G. B. Preston, The algebraic theory of semigroups, Vol. 1, Math. Surveys of the Amer. Math. Soc., 7 (Providence, R.I., 1961).

3. J. Lambek, Lectures on rings and modules (Blaisdell, 1966).

4. F. R. McMorris, On quotient semigroups; submitted.

5. Y. Utumi, On quotient rings, Osaka Math. J. 8 (1956), 1-18.

Bowling Green State University

Bowling Green, Ohio 43402 